\title{
VIII. On the second law of thermodynamics in connexion with the kinetic theory of gases
}

\section{S.H. Burbury}

To cite this article: S.H. Burbury (1876) VIII. On the second law of thermodynamics in connexion with the kinetic theory of gases, Philosophical Magazine Series 5, 1:1, 61-67, DOI: 10.1080/14786447608639002

To link to this article: http://dx.doi.org/10.1080/14786447608639002

曲 Published online: 13 May 2009.

Submit your article to this journal $\sqsubset x$

Џ Article views: 3

Q View related articles $\square$

Citing articles: 1 View citing articles $\square$ 


\section{$\left[\begin{array}{ll}61 & ]\end{array}\right.$}

VIII. On the Second Law of Thermodynamics in connexion with the Kinetic Theory of Gases. By S. H. BuRBury *.

TN a memoir by Boltzmann $\dagger$ is contained an analytical 1 proof of the second law of thermodynamics, founded on results obtained in the earlier part of the same memoir. The writer's treatment of this part of the subject is not characterized by his usual lucidity, and has given much trouble to some mathematicians. It is worth while to show that the second law can be deduced by a simple process from Boltzmann's results.

A great number, $\lambda$, of material particles are moving in a closed spherical vessel. Forces of mutual attraction or repulsion act between each pair of particles and between the particles and the walls of the vessel. To simplify the case, we will suppose that these are the only forces which act on the particles.

Boltzmann has shown that the mean kinetic energy is the same for every particle. Let $T$ be this mean kinetic energy, and let $\mathrm{T}=\frac{3}{2} \bar{h}$. The mean kinetic enegy of the system is then $\frac{3 \lambda}{2 h}$.

Let the positions of the particles at any instant be defined by reference to three rectangular axes passing through the centre of the vessel, the coordinates of the several particles being $x_{1}, y_{1}, z_{1}, \ldots x_{\lambda}, y_{\lambda}, z_{\lambda}$.

Let $v$ be the volume of the vessel.

The pressure exerted by the system of moving particles on any point on the surface of the sphere may vary from instant to instant according to the positions which the particles happen to have. But if the motion be stationary, this pressure must have on the average of any very long time a certain mean value $P$ per unit area, which in the absence of impressed forces will be uniform over the whole surface.

Then $P$ is the force per unit area which must be exerted on the surface of the sphere from without in order to maintain equilibrium.

Let $\chi$ be the ergal for any given positions of the particles of all the forces acting on the particles-that is, of all the forces which act in any manner on the system, except the external force $P$.

All those positions of the particles for which the coordinates of the first particle are between $x_{1}$ and $x_{1}+d x_{1}$,

$$
\begin{aligned}
& y_{1} \text { and } y_{1}+d y_{1}, \\
& z_{1} \text { and } z_{1}+d z_{1},
\end{aligned}
$$

* Communicated by the Author.

$\dagger$ Sitzungsberichte der Wiener Akad. vol. Ixiii. 
62 Mr. S. H. Burbury on the Second Law of Thermodynamics of the second particle between $x_{2}$ and $x_{2}+d x_{2}$, $y_{2}$ and $y_{2}+d y_{2}$

- . . . . ,

of the $\lambda$ th particle between $\quad x_{\lambda}$ and $x_{\lambda}+d x_{\lambda}$, $y_{\lambda}$ and $y_{\lambda}+d y_{\lambda}$,

$z_{\lambda}$ and $z_{\lambda}+d z_{\lambda}$,

may, when $d x_{1}, d y_{1}, \ldots d z_{\lambda}$ are very small, be considered as having the same value of $\chi$ for ergal, notwithstanding any variations of position within those limits.

Let all such positions be deemed to constitute one configuration, which we may call configuration $M$. Then, as Boltzman has shown, the time during which on the average of any very long time the system will be within configuration $M$ is proportional to

$$
\overline{\boldsymbol{\epsilon}}^{h_{\mathrm{x}}} d x_{1} d y_{1} \ldots d z_{\lambda}
$$

Its actual value is therefore

$$
\frac{\bar{\epsilon}^{h x} d x_{1} d y_{1} \ldots d z_{\lambda}}{\iiint \ldots \bar{\epsilon}^{\hbar x} d x_{1} d y_{1} \ldots d z_{\lambda}}
$$

the integration extending, over all possible configurations.. The mean value of any function, $f$, of the position of the particles (which we denote as usual by $\bar{f}$ ) is

and therefore

$$
\bar{f}=\iiint \int_{\int \bar{\epsilon}^{h} x} d x_{1} d y_{1} \ldots d z_{\lambda}
$$

$$
\bar{\chi}=\frac{\iiint \ldots \bar{\chi}^{\bar{\epsilon}^{h x}} d x_{1} d y_{1} \ldots d z_{\lambda}}{\iiint \ldots \bar{\epsilon}^{h x} d x_{1} d y_{1} \ldots d z_{\lambda}}
$$

II. Now let the system receive a small accession of heat, $\delta Q$, and let $\delta h, \delta v$ be the consequent increments of $h$ and $v$. We may suppose the alteration of volume to be effected by the vessel undergoing a uniform homogeneous strain, the centre remaining fixed. In that case the distance, $r$, between any two points in it beomes after the alteration $\theta r$, where $\theta$ is a ratio the same for every pair of points, and manifestly $\theta=1+\frac{1}{3} \frac{\delta v}{v}$.

We further assume that the external forces are so adjusted as to maintain the system in equilibrium in its altered state, and that such adjustment takes place without altering $\chi$ for any given configuration, consequently that no change takes 
place in $\chi$ except as the equivalent of work done by or against the forces on change of position of the particles. Then $\delta Q$ consists (1) of alteration of kinetic energy-that is,

$$
\delta\left(\frac{3 \lambda}{2 h}\right) \text { or }-\frac{3 \lambda}{2 h^{2}} \delta h ;
$$

(2) of alteration $\delta \bar{\chi}$ of $\bar{\chi}$; (3) of external work done, P $\delta v$ - that is,

$$
\delta Q=-\frac{3 \lambda}{2 h^{2}} \delta h+\delta \bar{\chi}+P \delta v .
$$

We may here observe that

$$
\delta \mathrm{Q}-\mathrm{P} \delta v=-\frac{3 \lambda}{2 h^{2}} \delta h+\delta \bar{\chi}
$$

which is an exact differential of a function of two variables, $h$ and $v$. This proposition is proved in a different way by Rankine (see 'The Steam-Engine,' eighth edition, pp. 304-313). Again,

$$
\frac{\delta \mathrm{Q}}{\mathrm{T}}=-\frac{\lambda}{h} \delta h+\frac{2 h}{3} \delta \vec{\chi}+\frac{2 h}{3} \mathrm{P} \delta v ; \quad . \quad .
$$

and it is required to prove that this expression is an exact differential.

III. Consider the expression

$$
\log \iiint . . \overline{\boldsymbol{\epsilon}}^{h \mathbf{x}} d x_{1} d y_{\mathbf{1}} \ldots d z_{\lambda}
$$

in which the integration extends over all configurations ; and let

$$
\log \iiint \ldots \overline{\boldsymbol{\epsilon}}^{h x} d x_{1} d y_{1} \ldots d z_{\lambda}=u \text {. }
$$

Then $\delta u$ denotes the whole change which takes place in $u$ consequent on $h$ becoming $h+\delta h$, and $v$ becoming $v+\delta v$; that is,

$$
\delta u=\frac{d u}{d h} \delta h+\frac{d u}{d v} \delta v .
$$

Now for any given configuration the ergal $\chi$ is not altered by alteration of $h$, though the comparative frequency of the occurrence of such configuration is altered; therefore

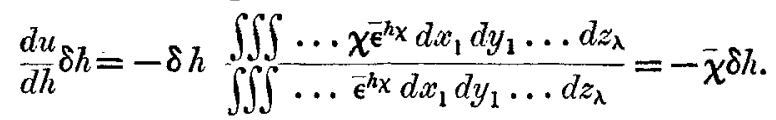

We have now to find $\frac{d u}{d v} \delta v$. Both in the original and in the altered volume the integration is to be extended over all possible configurations. Now for every configuration $M$ in the original volume there is in the altered volume a correspond- . ing configuration, which we may call $\mathrm{M}^{\prime}$; and $\mathbf{M}^{\prime}$ is to be 
$64 \mathrm{Mr}$. S. H. Burbury on the Second Law of Thermodynamics

formed from $\mathrm{M}$ by altering the distance between each pair of points in the ratio $\theta: 1$-that is,

$$
\left\{1+\frac{1}{3} \frac{\delta v}{v}\right\}: 1 \text {. }
$$

If, then, $u, \chi, d x_{1}, d y_{1}, \ldots d z_{\lambda}$ be denoted in the altered volume by accented letters, we shall have

$$
\begin{aligned}
u^{\prime}=\log \iiint \ldots \bar{\epsilon}^{h x^{\prime}} d x_{1}^{\prime} d y_{1}^{\prime} \ldots d z_{\lambda}^{\prime} \\
\\
=\log \left\{\theta^{3 \lambda} \bar{\epsilon}^{h x^{\prime}} d x_{1} d y_{1} \ldots d z_{\lambda}\right\},
\end{aligned}
$$

since every linear element $d x_{1}, d y_{1}, \& c$. is altered in the ratio $\theta: 1$.

In the last expression for $u^{\prime}, \chi^{\prime}$ is to be treated as a function of $x_{1}, y_{1}, \ldots z_{\lambda}$. Then

$$
\begin{aligned}
\frac{\delta u}{d v} \delta v & =u^{\prime}-u=\log \frac{\iiint \ldots \bar{\epsilon}^{h x^{\prime}} d x^{\prime}{ }_{1} d y^{\prime}{ }_{1}{ }^{\prime} \ldots d z_{\lambda}^{\prime}}{\iiint \ldots \bar{\epsilon}^{h x} d x_{1} d y_{1} \ldots d z_{\lambda}} \\
& =\log \left\{\theta^{3 \lambda} \frac{\iiint \ldots \bar{\epsilon}^{h x^{\prime}} d x_{1} d y_{1} \ldots d z_{\lambda}}{\iiint \ldots \bar{\epsilon}^{h x} d x_{1} d y_{1} \ldots d z_{\lambda}}\right\} \\
& =3 \lambda \log \theta-h \cdot \frac{\iiint \ldots \delta \chi^{h} d x_{1} d y_{1} \ldots d z_{\lambda}}{\iiint \ldots \bar{\epsilon}^{h x} d x_{1} d y_{1} \ldots d z_{\lambda}} \\
& =\lambda \frac{\delta v}{v}-h \delta v \cdot \frac{\iiint \ldots \frac{d \chi}{d v} \bar{\epsilon}^{h x} d x_{1} d y_{1} \ldots d z_{\lambda}}{\iiint \ldots \bar{\epsilon}^{h x} d x_{1} d y_{1} \ldots d z_{\lambda}} \\
& =\lambda \frac{\delta v}{v}-h \frac{\overline{d x}_{d v} \delta v}{}
\end{aligned}
$$

in which expression $\frac{\overline{d \chi}}{d v}$ denotes the mean for all configurations of the alteration of $\chi$ consequent on $v$ becoming $v+\delta v$.

Collecting our results, we obtain

$$
\delta u=\frac{d u}{d h} \delta h+\frac{d u}{d v} \delta v=-\bar{\chi} \delta h+\lambda \frac{\delta v}{v}-h \frac{\overline{d \chi}}{d v} \delta v . \quad .
$$

IV. The system being in stationary motion must satisfy Clausius's equation

$$
\frac{3}{2} \mathrm{P} v=\frac{3 \lambda}{2 h}+\frac{1}{2} \overline{\Sigma \Sigma R} r
$$

in which $\mathrm{P}$ is the force acting from without on the surface of the vessel, and $\frac{1}{2} \overline{\Sigma \Sigma \mathrm{R} r}$ is the mean virial of all the other forces acting on the system-that is, of all the forces of which the ergal is $\chi$. 
in connexion with the Kinetic Theory of Gases.

Also in $\Sigma \Sigma R r, \mathrm{R}$ is the repulsive force, $r$ the distance between any two particles, or between a particle and a point on the surface, and the summation includes every pair of points between which any force acts.

Then $\Sigma \Sigma R r$ is a function of position of the particles, having a definite value for each configuration, and $\Sigma \Sigma \overline{R r}$ denotes the mean value of $\Sigma \Sigma R r$ for any long time; and we might therefore write

$$
\overline{\Sigma \Sigma} \mathrm{R} r=\frac{\iiint \ldots(\Sigma \Sigma \mathrm{R} r) \bar{\epsilon}^{h_{\chi}} d x_{1} d y_{1} \ldots d z_{\lambda}}{\iiint \ldots \bar{\epsilon}^{h \chi} d x_{1} d y_{1} \ldots d z^{\lambda}} .
$$

Now let

and let

$$
\mathrm{P}=\mathrm{P}_{1}+\mathrm{P}_{2}
$$

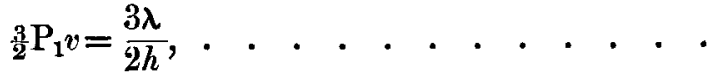

$$
\begin{aligned}
& \frac{3}{2} \mathrm{P}_{\mathrm{q}} v=\frac{1}{2} \overline{\Sigma \Sigma \mathrm{R} r} \text {. . . . . . . . . . . }
\end{aligned}
$$

It follows from (1) that

and

$$
h \mathrm{P}_{1}=\frac{\lambda}{v}
$$

$$
h \mathrm{P}_{1} \delta v=\lambda \frac{\delta v}{v} .
$$

Also from (2),

$$
\begin{aligned}
\mathrm{P}_{2} & =\overline{\frac{1}{3} \Sigma \Sigma \mathrm{R} \frac{r}{v},} \\
\mathrm{P}_{2} \delta v & =\frac{1}{3 \Sigma \Sigma \mathrm{R} \frac{\delta v}{v} r} \\
& =\overline{\Sigma \Sigma \mathrm{R} \delta r .}
\end{aligned}
$$

Now for each configuration

$$
\Sigma \Sigma \mathrm{R} \delta r=-\delta \chi=-\frac{d \chi}{d v} \delta v
$$

Therefore, taking mean values,

therefore

$$
\overline{\Sigma \Sigma K \delta}=-\frac{\overline{d \chi}_{d}}{d v} \delta
$$

Therefore

$$
\mathrm{P}_{2} \delta v=\overline{\Sigma \Sigma R \delta r}=-\frac{\overline{d \chi}}{d v} \delta v
$$

$$
h \mathrm{P}_{\mathbf{z}} \delta v=-h \frac{\overline{d \chi}_{\bar{\chi}}}{d v} \text {. }
$$

Phil. Mag. S. 5. Vol, 1, No. 1. Jan. 1876. 
And we have seen that

Therefore

$$
h \mathrm{P}_{\mathrm{t}} \delta v=\lambda \frac{\delta v}{v} .
$$

that is,

$$
h \mathrm{P}_{1} \delta v+h \mathrm{P}_{2} \delta v=\lambda \frac{\delta v}{v}-h \frac{\overline{d \chi}}{d v} \delta v
$$

$$
h \mathrm{P} \delta v=\lambda \frac{\delta v}{v}-h \frac{\overline{d \chi}}{d v} \delta v .
$$

Substituting in $(\mathrm{B})$, we obtain

or

$$
\delta u=-\bar{\chi} \delta h+h \mathrm{P} \delta v
$$

$$
\hbar \mathrm{P} \delta v=\bar{\chi} \delta / \iota+\delta u
$$

and substituting in (A), we obtain

$$
\begin{array}{r}
\frac{\delta \mathrm{Q}}{\mathrm{T}}=-\lambda \frac{\delta h}{h}+\frac{2}{3} h \delta \bar{\chi}+\frac{2}{3} \bar{\chi} \delta h+\frac{2}{3} \delta u \\
=\delta\left\{-\lambda \log h+\frac{2}{3} h \bar{\chi}+\frac{2}{3} u\right\},
\end{array}
$$

an exact differential.

The expression $-\lambda \log h+\frac{2}{3} h \bar{\chi}+\frac{2}{3} u$ is what Rankine denotes by $\phi$ in his equation

$$
\mathrm{J} d \mathrm{Q}=\mathrm{T} d \phi \text {. }
$$

The value which we have obtained for $\phi$ is the same as that obtained by Boltzmann in the memoir above referred to. But his expression for $\delta Q$ is

$$
\begin{aligned}
& -\frac{3 \lambda}{2 h^{2}} \delta h+\delta\left\{\frac{\iiint \ldots \chi^{-\epsilon^{h x}} d x_{1} d y_{1} \ldots d z \lambda}{\iiint \ldots \bar{\epsilon}^{h x} d x_{1} d y_{1} \ldots d z_{\lambda}}\right\} \\
& -\frac{\iiint \ldots \delta \chi \overline{\mathrm{e}}^{\bar{h}_{\chi}} d x_{1} d y_{1} \ldots d \dot{z}_{\lambda}}{\iiint \ldots \overline{\boldsymbol{\epsilon}}^{h x} d x_{1} d y_{1} \ldots d z_{\lambda}},
\end{aligned}
$$

which is not easily to be reconciled with the expression we have used.

$V$. We have assumed hitherto that no alteration takes place in $\chi$ except as the equivalent of work done by or against the forces. It is, however, conceivable that a change should take place in $\chi$ for one and the same configuration, especially if, in the adjustment of external pressure to maintain equilibrium, new forces be brought into play. Boltzmann appears to contemplate this case. We proceed to show that, provided $\boldsymbol{x}$ be for each 
configuration a function of $h$ and $v$, the function $\frac{d \mathrm{Q}}{\mathrm{T}}$ is still an exact differential.

Let $\delta_{2} \chi$ or $\frac{d_{2} \chi}{d h} \delta h+\frac{d_{2} \chi}{d v} \delta v$ be the new alteration in $\chi$, which is not the equivalent of work done, and let $\delta_{2} \bar{\chi}$ be the mean value of $\delta_{2} \chi$.

Then, as the alteration is supposed to take place without expense to $\delta Q_{1}, \delta_{2} \bar{\chi}$ must be subtracted from the whole change of the ergal in the expression for $\delta Q$. The equation (A) then becomes

$$
\frac{\delta \mathrm{Q}}{\mathrm{T}}=-\lambda \frac{\delta h}{h}+\frac{2}{3} h \delta \bar{\chi}-\frac{2}{3} h \delta_{2} \bar{\chi}+\frac{2}{3} h \mathrm{P} \delta r .
$$

Under the circumstances (B) becomes

$$
\begin{aligned}
\delta u & =-\bar{\chi} \delta h+\lambda \frac{\delta v}{v}-h \frac{\overline{d \chi}}{d v} \delta v-h \frac{\overline{d_{2} \chi}}{d h} \delta h-h \frac{\overline{d_{2} \chi}}{d v} \delta v \\
& =-\bar{\chi} \delta h+\lambda \frac{\delta v}{v}-h \frac{\overline{d \chi}}{d v} \delta v-h \delta_{2} \bar{\chi}
\end{aligned}
$$

and by substitution we obtain, as before,

$$
\frac{\delta Q}{\mathrm{~T}}=\delta\left\{-\lambda \log h+\frac{2}{3} h \bar{\chi}+\frac{2}{3} u\right\}=\delta \phi .
$$

IX. On the Production of Spectra by the Oxyhydrogen Flame. By Tasker H. Marvin, Brooklyn, N.Y."

QIX or seven months since, in using the lime-light for spec$S$ troscopic work, I had the good fortune to discover a method by which bright-line spectra, without an accompanying continuous spectrum, could be produced at will by means of the oxyhydrogen flame.

I had been engaged in examining the spectrum of calcium superimposed upon the continuous spectrum, which is easily seen by bringing the collimator of a compound spectroscope to bear laterally upon the incandescent point of the line. I found that I could bring out all the bands of calcium and the sodium line doubled sharply and brightly, notwithstanding the brightness of the continuous spectrum. When, however, the proportions of hydrogen and oxygen were so adjusted that there was no excess of the latter, the bright lines of calcium and sodium disappeared and the continuous spectrum alone remained. The chemical reaction then seemed evident; the

* Communicated by the Authox.

F 2 